\title{
Chern-Simons theory, exactly solvable models and free fermions at finite temperature
}

\author{
Miguel Tierz \\ Volen Center for Complex Systems, Brandeis University, 415 South Street, Waltham, \\ MA 02454-9110, USA. E-mail: tierz@brandeis.edu
}

\begin{abstract}
We show that matrix models in Chern-Simons theory admit an interpretation as 1D exactly solvable models, paralleling the relationship between the Gaussian model and the Calogero model. We compute the corresponding Hamiltonians, ground-state wavefunctions and ground-state energies and point out that the models can be interpreted as quasi-1D Coulomb plasmas. We also study the relationship between Chern-Simons theory on $S^{3}$ and a system of $\mathrm{N}$ one-dimensional fermions at finite temperature with harmonic confinement. In particular we show that the Chern-Simons partition function can be described by the density matrix of the free fermions in a very particular, crystalline, configuration. For this, we both use the Brownian motion and the matrix model description of Chern-Simons theory and find several common features with $\mathrm{c}=1$ theory at finite temperature. Finally, using the exactly solvable model result, we show that the finite temperature effect can be described with a specific two-body interaction term in the Hamiltonian, with 1D Coulombic behavior at large separations.
\end{abstract}




\section{Introduction}

In addition to its original remarkable impact in topology, Chern-Simons theory [1] has also enjoyed a considerable interest in high-energy physics and condensed matter physics alike (e.g. in the fractional quantum Hall effect [2]). Recall that pure ChernSimons theory is characterized by an action

$$
S_{\mathrm{CS}}(A)=\frac{k}{4 \pi} \int_{M} \operatorname{Tr}\left(A \wedge d A+\frac{2}{3} A \wedge A \wedge A\right),
$$

with $k$ an integer number. Chern-Simons theory provides a physical approach to three dimensional topology. In particular, it gives three-manifold invariants and knot invariants. For example, the partition function,

$$
Z_{k}(M)=\int \mathcal{D} A \mathrm{e}^{i S_{\mathrm{CS}}(A)}
$$

delivers a (quantum) topological invariant of $M$. In recent years there has been a considerable amount of interest in pure Chern-Simons theory due to its role in topological strings [3].

Chern-Simons theory on certain manifolds is also known to have a simple description in terms of random matrix theory [4, 5]. In this work, we shall employ this relationship to present a simple description of Chern-Simons theory in terms of a one-dimensional plasma with long-range Coulombic interactions. More precisely, we shall show that simple one-component and two-component plasma models, with a two-body interacting term that is a Coulomb potential of restricted dimension 1 , describes Chern-Simons theory on $S^{3}$ and on lens spaces (to a certain extent). This happens in the following simple way: the wavefunctional that appears in the canonical quantization of ChernSimons theory, can be reduced to an explicit many-body ground state wavefunction whose Hamiltonian, which only contains one-body and two-body potentials, is explicitly found. We also study the relationship between Chern-Simons theory on $S^{3}$ and a system of $N$ one-dimensional fermions at finite temperature with harmonic confinement. In particular, we show that the partition function of Chern-Simons theory can be described by the density matrix of the free fermions in a very particular configuration.

Let us now briefly summarize the precise relationship between Chern-Simons theory and random matrix models, a result that we shall employ in the following. Lawrence and Rozansky, in several works [6, 7], using the exact, non-perturbative, expressions

\footnotetext{
${ }^{1}$ Restricted dimension means that while the model is in $1 d$, the potential is the one corresponding to another dimension. In our case, the surface of a $2 d$ cylinder.
} 
for Chern-Simons theory found by Witten [1], have shown that the partition function of Chern-Simons theory on Seifert manifolds has a simple structure and that this partition function can be expressed entirely as a sum of local contributions from the flat connections on the manifold. The contribution may come from reducible and, for certain manifolds that will not be discussed here, also irreducible flat connections. The reducible flat connections can be expressed as an integral, instead of the complex sums over integrable representations of the affine Lie algebra associated with the gauge group of Chern-Simons theory [1]. The irreducible flat connections contributions are given by residue terms but for the two simplest manifolds in the Seifert family, the ones that we discuss here, $S^{3}$ and lens spaces, only reducible flat connections contribute. In the simplest case, that of $S^{3}$, the trivial flat connection contribution gives the full partition function. See [4, 8] and references therein for more details.

The connection with random matrix theory [9] comes from the integral representation of the contribution of reducible flat connections. In particular, in [4], the work of Lawrence and Rozansky is extended from $S U(2)$ to generic simply-laced gauge groups, like $U(N)$ or $O(2 N)$ and, in addition, by specifying an orthonormal basis and expanding the Dynkin coordinates and the positive roots of the Cartan subalgebra of the gauge group in terms of this basis, concrete $N$-dimensional integral expressions, reminiscent of random matrix theory are found. Let us consider the simplest possible case, that of gauge group $U(N)$ and a $S^{3}$ manifold 2 [4, 5, 10]

$$
Z_{\mathrm{CS}}\left(S^{3}\right)=\frac{\mathrm{e}^{-\frac{1}{12} N\left(N^{2}-1\right) g_{s}}}{N !} \int \prod_{i=1}^{N} \frac{\mathrm{d} \lambda_{i}}{2 \pi} \mathrm{e}^{-|\lambda|^{2} / 2 g_{s}} \prod_{i<j}\left(2 \sinh \frac{\lambda_{i}-\lambda_{j}}{2}\right)^{2} .
$$

or, in matrix space [5, 10]

$$
Z_{\mathrm{CS}}\left(S^{3}\right)=\frac{\mathrm{e}^{-\frac{1}{12} N\left(7 N^{2}-1\right) g_{s}}}{N !}\left(\frac{2 \pi}{g_{s}}\right)^{-N / 2} \int[d M] \mathrm{e}^{-\frac{1}{2 g_{s}} \operatorname{Tr}(\log M)^{2}},
$$

which are related by a simple exponential mapping [5, 11]. In this last form, it can be explicitly computed with the associated Stieltjes-Wigert orthogonal polynomials [5], giving the known value [1]

$$
Z_{\mathrm{CS}}\left(S^{3}\right)=\frac{\mathrm{e}^{\frac{i \pi N^{2}}{4}}}{(k+N)^{N / 2}} \prod_{j=1}^{N-1}\left(2 \sin \frac{\pi j}{k+N}\right)^{j-N}
$$

It is well-known that the Wigner-Dyson distribution of eigenvalues $P_{N}^{\beta}\left(\left\{\lambda_{i}\right\}\right)$ coincides with the probability distribution of the $N$-particle coordinates $P_{N}^{\beta}\left(\left\{r_{i}\right\}\right)$ of the quantum

\footnotetext{
${ }^{2}$ The coupling constant $g_{s}$ is related with the $k$ level of Chern-Simons theory by $g_{s}=\frac{2 \pi i}{k+N}[4$, 5]
} 
ground state of a $1 D$ Hamiltonian: the Calogero model. When there is a harmonic confinement term, the ground state wavefunction correspond to the Gaussian random matrix ensembles. The same property holds true for the Chern-Simons matrix model (1.3) , as we shall show and discuss in detail in the next Section.

\section{$2 \quad 1 D$ exactly solvable model}

Thus, the natural question, not yet addressed, is whether one can consider:

$$
\Psi_{0}\left(x_{1}, \ldots, x_{N}\right)=\sqrt{\alpha_{N}} \prod_{i=1}^{N} \mathrm{e}^{-\frac{x_{i}^{2}}{2 g_{s}}} \prod_{i<j}\left(\sinh \frac{x_{i}-x_{j}}{2 L}\right)^{m},
$$

so that

$$
Z_{N}=\left\langle\Psi_{0} \mid \Psi_{0}\right\rangle, \quad \text { with } L=m=1
$$

with

$$
H \Psi_{0}=E_{0} \Psi_{0}, \quad H=-\sum_{i=1}^{N} \frac{d^{2}}{d x_{i}^{2}}+\sum_{i=1}^{N} V\left(x_{i}\right)+\sum_{i<j} W\left(x_{i}-x_{j}\right) .
$$

In principle, according to [12], (2.1) should not correspond to an exactly solvable model satisfying (2.3) (like Calogero model for example). A careful treatment of (2.3) with a generic wavefunction

$$
\Psi_{0}\left(x_{1}, \ldots, x_{N}\right)=\prod_{i=1}^{N} \sigma\left(x_{i}\right) \prod_{1 \leq i<j \leq N} \chi\left(x_{i}-x_{j}\right),
$$

shows that there are more exactly solvable models than has been recognized hitherto. This has been carried out in [13], but this work has gone largely unnoticed. It is noteworthy that the Chern-Simons model is a representative of this class of Hamiltonians and already a simple computation (namely, applying the operator $-\sum \frac{d^{2}}{d x_{i}^{2}}$ in (2.1)) shows it. This fact was already pointed out in [14] (as a counterexample to the list of exactly solvable models provided in [12]). Therefore, one has [14]

$$
\begin{aligned}
H & =-\sum_{i=i}^{N} \frac{\partial^{2}}{\partial x_{i}^{2}}+\frac{1}{g_{s}^{2}} \sum_{i=1}^{N} x_{i}^{2}+\frac{m}{g_{s} L} \sum_{i<j}\left(x_{i}-x_{j}\right) \operatorname{coth}\left(\frac{x_{i}-x_{j}}{2 L}\right)+\frac{m(m-1)}{2 L} \sum_{i<j} \frac{1}{\sinh ^{2}\left(\frac{x_{i}-x_{j}}{2 L}\right)} \\
E_{0} & =-\frac{m^{2}}{3}\left(\frac{1}{2 L}\right)^{2} N(N-1)(N-2)+\frac{N}{g_{s}}-\left(\frac{1}{2 L}\right)^{2} m^{2} N(N-1) .
\end{aligned}
$$

The computation is instructive since the model has rather simple properties. The only apparent complication are possible three-body terms. However, they always cancel 
in groups of three and contribute to the energy of the ground state with the term $\frac{1}{3} N(N-1)(N-2)$, the leading term in $E_{0}$.

Indeed, the problem (2.3) with (2.4) amounts to the solution of a certain functional equation whose traditional solution (that lead to, among others, the Calogero model) is shown in [13] to be incomplete. More precisely, for (2.3) with a generic wavefunction: two different functional equations have to be solved. The first functional equation is the one that appears when $\sigma(x)=1$ :

$$
\varphi(x) \varphi(y)+\varphi(y) \varphi(z)+\varphi(z) \varphi(x)=f(x)+f(y)+f(z) \quad \text { for } \quad x+y+z=0,
$$

with $\varphi(x)=\chi^{\prime}(x) / \chi(x)$. The second functional equation appears because applying (2.3) to (2.4) leads to a term $\sum_{i \neq j} \frac{1}{2} \varphi\left(x_{i}-x_{j}\right)\left(\tau\left(x_{i}\right)-\tau\left(x_{j}\right)\right)$, where $\varphi(x)$ is defined as above and $\tau(x)=\sigma^{\prime}(x) / \sigma(x)$, that has to be expressed in terms of one-body or two-body potentials in order to achieve exact solvability. But it turns out that what has been usually considered is the functional equation:

$$
\varphi\left(x_{i}-x_{j}\right)\left(\tau\left(x_{i}\right)-\tau\left(x_{j}\right)\right)=\lambda(x)+\lambda(y) .
$$

However, as correctly pointed out in [13], a more general consideration naturally leads to:

$$
\varphi\left(x_{i}-x_{j}\right)\left(\tau\left(x_{i}\right)-\tau\left(x_{j}\right)\right)=\lambda(x)+\lambda(y)+F(x-y) .
$$

The cancellations above discussed are due to the fact that for the Chern-Simons models $f(x)$ in (2.6) is a constant. It is also a simple to see that $\lambda(x)=0$ and that $F(x-y)=$ $(x-y) \operatorname{coth}(x-y)$.

To neglect $F(x-y)$ amounts to study the possible models that can explain an addition or modification in the confining (one-body) part of the ground state wavefunction (2.4) only with an addition or modification of the confining (one-body) part of the Hamiltonian itself. These leaves a wealth of models where such aspect of the ground state wavefunction is described through a modification of the correlations of the many-body problem (that is, through a two-body potential in the Hamiltonian).

Notice that the model (2.1) can also be interpreted as an addition of a Gaussian one-body factor to the hyperbolic Sutherland model, at the level of the wavefunction. Then, it shares $f(x)$ with this model as this function only depends on the twobody part of the wavefunction (namely $\varphi(x)$ ). In addition, as explained just above, this modification can only be explained by the inclusion of additional interactions at the level of the Hamiltonian, which are given in this particular case by the term 
$\sum_{i<j}\left(x_{i}-x_{j}\right) \operatorname{coth}\left(\frac{x_{i}-x_{j}}{2 L}\right)$. Note also that in the Coulomb gas picture, the ground-state wavefunction is written:

$$
\Psi_{0}=\mathrm{e}^{-\mathcal{H}}, \quad \mathcal{H}=\frac{1}{2 g_{s}} \sum_{i=1}^{N} x_{i}^{2}-\sum_{i<j} \ln \sinh \left|\frac{x_{i}-x_{j}}{2 L}\right| .
$$

The last interacting term is known to be the Coulomb potential between charges in the surface of a $2 D$ cylinder [14]. At small distances $\left|x_{i}-x_{j}\right| \ll L$, the interaction term in (2.9) behaves like the $2 D$ Coulomb interaction $V\left(x_{i}-x_{j}\right)=\ln \left|x_{i}-x_{j}\right|$, while at large distances along the cylinder it behaves like the $1 D$ Coulomb interaction $V\left(x_{i}-x_{j}\right)=$ $\left|x_{i}-x_{j}\right|$. The radius of the cylinder is an additional length that conspires with $g_{s}$, the dimensionful parameter in the quadratic confining potential, and gives rise to the dimensionless $q$-parameter $q=\mathrm{e}^{-\frac{g_{s}}{L}}$, which corresponds to the usual $q$-parameter in Chern-Simons theory [5] (recall the model derived in Chern-Simons theory leads to a fixed radius for the cylinder $L=1$ ). A simple mapping shows this explicitly [5, 10, 14], while it allows the model to be solved exactly in terms of $q$-orthogonal polynomials. This parameter is the responsible for the discrete scale invariance of the model [10]. In particular, the model is known to posses Wigner solid behavior [14] (see also plots in [10]). It is worth to mention that the two-body term can also be easily interpreted as a one-dimensional crystal potential with transverse periodic boundary condition 1 . Another strong indication of crystalline behavior comes from a result in [10], where it was shown that the model can be discretized (with an homogeneous lattice), without modifying its meaning in Chern-Simons theory.

The result goes beyond the gauge group and geometry above considered and, for example, from [4] we can readily suggest analogous expressions for orthogonal:

$$
\Psi_{0}^{S O(2 N)}\left(x_{1}, \ldots, x_{N}\right)=\prod_{i=1}^{N} \mathrm{e}^{-\frac{x_{i}^{2}}{2 g_{s}}} \prod_{i<j} \sinh ^{m}\left(\frac{x_{i}-x_{j}}{2 L}\right) \sinh ^{m}\left(\frac{x_{i}+x_{j}}{2 L}\right),
$$

and symplectic group:

$$
\Psi_{0}^{S p(2 N)}\left(x_{1}, \ldots, x_{N}\right)=\prod_{i=1}^{N} \mathrm{e}^{-\frac{x_{i}^{2}}{2 g_{s}}} \sinh \left(\frac{x_{i}}{L}\right) \prod_{i<j} \sinh ^{m}\left(\frac{x_{i}-x_{j}}{2 L}\right) \sinh ^{m}\left(\frac{x_{i}+x_{j}}{2 L}\right),
$$

again with $m=L=1$ in Chern-Simons theory. Computations are slightly more involved, as there are more apparent three-body terms, but they cancel in exactly the

\footnotetext{
${ }^{1}$ This result is immediate from basic results on crystal potentials [15] and the elementary Fourier series $\sum_{n=1}^{\infty} \frac{1}{n} \cos n x=-\ln \left[2 \sin \left(\frac{|x|}{2}\right)\right]$
} 
same way, leading to:

$$
\begin{aligned}
H_{S O(2 N)} & =-\sum_{i=i}^{N} \frac{\partial^{2}}{\partial x_{i}^{2}}+\frac{1}{g_{s}^{2}} \sum_{i=1}^{N} x_{i}^{2}+\frac{m}{g_{s} L} \sum_{i<j}\left(\left(x_{i}-x_{j}\right) \operatorname{coth}\left(\frac{x_{i}-x_{j}}{2 L}\right)+\left(x_{i}+x_{j}\right) \operatorname{coth}\left(\frac{x_{i}+x_{j}}{2 L}\right)\right) \\
& +\frac{m(m-1)}{2 L} \sum_{i<j}\left(\frac{1}{\sinh ^{2}\left(\frac{x_{i}-x_{j}}{2 L}\right)}+\frac{1}{\sinh ^{2}\left(\frac{x_{i}+x_{j}}{2 L}\right)}\right) . \\
E_{0} & =-\frac{4}{3}\left(\frac{1}{2 L}\right)^{2} m^{2} N(N-1)(N-2)+\frac{N}{g_{s}}-2\left(\frac{1}{2 L}\right)^{2} m^{2} N(N-1) .
\end{aligned}
$$

for the orthogonal, and:

$$
\begin{aligned}
H_{S p(2 N)} & =-\sum_{i=i}^{N} \frac{\partial^{2}}{\partial x_{i}^{2}}+\frac{1}{g_{s}^{2}} \sum_{i=1}^{N} x_{i}^{2}+\frac{m}{g_{s} L} \sum_{i<j}\left(\left(x_{i}-x_{j}\right) \operatorname{coth}\left(\frac{x_{i}-x_{j}}{2 L}\right)+\left(x_{i}+x_{j}\right) \operatorname{coth}\left(\frac{x_{i}+x_{j}}{2 L}\right)\right) \\
& -\frac{2}{g_{s}} \sum_{i=1}^{N} x_{i} \operatorname{coth} \frac{x_{i}}{L}+\frac{m(m-1)}{2 L} \sum_{i<j}\left(\frac{1}{\sinh ^{2}\left(\frac{x_{i}-x_{j}}{2 L}\right)}+\frac{1}{\sinh ^{2}\left(\frac{x_{i}+x_{j}}{2 L}\right)}\right) \cdot(2.13) \\
E_{0} & =-\frac{4}{3}\left(\frac{1}{2 L}\right)^{2} m^{2}(N+1) N(N-1)+N\left(\frac{1}{g_{s}}-1\right)-2\left(\frac{1}{2 L}\right)^{2} m^{2} N(N-1) .
\end{aligned}
$$

for the symplectic case. In contrast to (2.1), these models have not been discussed elsewhere. As mentioned above, invariants other than the partition function can be considered. It is well-known that knot invariants can be obtained with [1]

$$
Z_{k}(M)=\int \mathcal{D} A W_{R}^{\mathcal{K}}(A) \mathrm{e}^{i S_{\mathrm{CS}}(A)},
$$

where $W_{R}^{\mathcal{K}}(A)$ is the Wilson loop operator, which is the trace of the holonomy around the knot. It is possible to generalize the results of [4] to other knot invariants [16]. For example, the case of torus knots is conjectured to be given by [17]

$$
W_{R}^{(P, Q)}=C_{N}(P, Q) \int \prod_{i=1}^{N} d x_{i} S_{\lambda_{\ell}}\left(\mathrm{e}^{x_{i}}\right) \mathrm{e}^{-\frac{x_{i}^{2}}{2 g_{s}}} \prod_{i<j}\left(2 \sinh \frac{x_{i}-x_{j}}{2 P}\right)\left(2 \sinh \frac{x_{i}-x_{j}}{2 Q}\right),
$$

where $\Lambda$ is the highest weight corresponding to the representation labelled by $\lambda$, and shifted by $\rho$ and $S_{\lambda}\left(x_{i}\right)$ are Schur polynomials associated to the partition $\lambda$.

The case $P=Q=1$ corresponds to the unknot. Then, the above integral reduces to the expression of the partition function of Chern-Simons theory with an insertion of a Schur polynomial $S_{\lambda}$. In this case, using the explicit expression for the Schur polynomial:

$$
S_{\lambda}\left(\mathrm{e}^{u}\right)=\frac{\sum_{w \in \mathcal{W}} \epsilon(w) \mathrm{e}^{\Lambda \cdot w(u)}}{\sum_{w \in \mathcal{W}} \epsilon(w) \mathrm{e}^{\rho \cdot w(u)}}
$$


together with Weyl's denominator formula,

$$
\sum_{w \in \mathcal{W}} \epsilon(w) \mathrm{e}^{w(\rho)}=\prod_{\alpha>0} 2 \sinh \frac{\alpha}{2}
$$

the above integral (2.15) reduces to a Gaussian, and can be performed exactly. One gets in fact:

$$
W_{\Lambda}(\text { unknot })=\frac{S_{\rho \Lambda}}{S_{\rho \rho}}
$$

and $W_{\Lambda}$ (unknot) is $\operatorname{dim}_{q} \lambda$, the quantum dimension of $\Lambda$. Thus, employing the groundstate wavefunctions one can write for the quantum dimensions

$$
\operatorname{dim}_{q} \lambda=\left\langle\Psi_{0}\left|S_{\lambda}\left(\mathrm{e}^{x}\right)\right| \Psi_{0}\right\rangle
$$

In [16], we give an explicit computation, using a mixture of combinatorial and orthogonal polynomials methods, of the integral that appears in the r.h.s. of (2.19) .

A more detailed study is clearly required in order to see if we can extend the description to excited states. For example, to see if (2.19) can be understood as an scalar product of excited states of the many-body Hamiltonian. For the moment, let us just show that other, more complex models, that appear in the literature, for example when one considers lens spaces, exhibit the same properties. We just present the result when the geometry is $S^{3} / \mathbb{Z}_{2}$, a case of interest in topological string theory (see [3] for a review). If we consider the corresponding (two-)matrix model [18], then the wave function is

$$
\begin{aligned}
\Psi_{0}^{S^{3} / \mathbb{Z}_{2}}\left(x_{1}, \ldots, x_{N_{1}}, y_{1}, \ldots, y_{N_{2}}\right)= & \prod_{i=1}^{N_{1}} \mathrm{e}^{-\frac{x_{i}^{2}}{2 g_{s}}} \prod_{j=1}^{N_{2}} \mathrm{e}^{-\frac{y_{j}^{2}}{2 g_{s}}} \prod_{1 \leq i<j \leq N_{1}} \sinh \left(\frac{x_{i}-x_{j}}{2}\right) \times \\
& \prod_{1 \leq i<j \leq N_{2}} \sinh \left(\frac{y_{i}-y_{j}}{2}\right) \prod_{i, j} \cosh \left(\frac{x_{i}-y_{j}}{2}\right) .
\end{aligned}
$$

Remarkably enough, explicit computation shows that all the three-body terms cancel as before, leading to

$$
\begin{aligned}
H_{S^{3} / \mathbb{Z}_{2}} & =-\sum_{i=i}^{N_{1}} \frac{\partial^{2}}{\partial x_{i}^{2}}-\sum_{i=i}^{N_{2}} \frac{\partial^{2}}{\partial y_{i}^{2}}+\frac{1}{g_{s}^{2}}\left(\sum_{i=1}^{N_{1}} x_{i}^{2}+\sum_{i=1}^{N_{2}} y_{i}^{2}\right)+\frac{1}{g_{s} L} \sum_{1 \leq i<j \leq N_{1}}\left(x_{i}-x_{j}\right) \operatorname{coth}\left(x_{i}-x_{j}\right) \\
& +\frac{1}{g_{s} L} \sum_{1 \leq i<j \leq N_{2}}\left(y_{i}-y_{j}\right) \operatorname{coth}\left(y_{i}-y j\right)+\frac{1}{g_{s} L} \sum_{i, j}\left(x_{i}-y_{j}\right) \tanh \left(x_{i}-y_{j}\right) .
\end{aligned}
$$


Since the corresponding matrix model is now a two-matrix model we have found the correspondence with a two-component plasma rather than the one-component plasmas above discussed.

To summarize this Section, we have presented one and two-component Coulomb plasmas that are also solutions of the 1D Hamiltonian problem (2.3) (like Calogero models, for example), and that provide a rather simple many-body description of Chern-Simons theory, for several gauge groups and geometries, mimicking its ability to deliver quantum topological invariants.

\section{Free fermions at finite temperature}

As we have explained above, Chern-Simons theory on certain manifolds has a simple description in terms of random matrix theory [4, 5]. In this Section, we shall employ this relationship to point out a connection between Chern-Simons theory and free fermions at finite temperature. In the rich interplay between supersymmetric topological strings, $c=1$ non-critical bosonic strings, IIB superstrings and Chern-Simons theory, this is possibly the less well-known one: the relationship between Chern-Simons theory and $c=1$ theory. This was already mentioned in [19] as the weakest link in the manifold connections between the above mentioned theories and models. An early study of the relationship between Chern-Simons theory and free fermions appeared long ago in [20]. Most of the considerations in this Section amount to rearrangements of what is previously known in the literature, but this rearrangement may be illuminating as some connections have not been recognized hitherto.

Before proceeding, recall that the structure of the $\mathcal{N}=2$ topological strings parallels that of bosonic topological strings. In [21] it was shown that critical $\mathcal{N}=2$ topological strings are mapped into $c=1$ non-critical strings. More precisely, they showed that the $c=1$ non-critical string corresponding to a CFT on a circle at the self-dual radius is equivalent to a topological $\mathcal{N}=2$ theory at the conifold. A particular consequence of this is that the genus expansion of the free energy of the $c=1$ string at self-dual radius coincides with the same expansion for the free energy when $N=\infty$ of $S U(N)$ Chern-Simons on $S^{3}$ [21]. In general, the relationship between $c=1$ theory and ChernSimons theory, as the one we have just mentioned involves topological matrix models (see [22] for a review), while we shall discuss a free fermion picture which involves the double-scaled matrix models of $2 D$ quantum gravity. Regarding the relationship between these double-scaled models of $c=1$ matrix quantum mechanics and Chern- 
Simons theory, it is only known that the free energy, when the target space is a circle, is very similar to the nonperturbative part of Chern-Simons theory on $S^{3}$ and gauge group $U(N)$ (see [23] for a recent review and discussions).

The matrix model description reviewed in the Introduction is in turn intimately related to a Brownian motion description of Chern-Simons theory [24], which is based on non-intersecting random walkers. The particular process that is related to ChernSimons theory is that of $N$ vicious walkers on a line. Walkers are vicious [25] if they annihilate each other when they meet. If we denote their coordinates by $\lambda_{i}$, $i=1, \ldots, N$, they satisfy $\lambda_{1}>\lambda_{2}>\ldots>\lambda_{N}$. Alternatively, this process can be regarded as motion of a single particle in the fundamental Weyl chamber of $U(N)$. The particle starts moving at position $\mu_{i}$ satisfying $\mu_{1}>\mu_{2}>\ldots>\mu_{N}$, and is required to stay within the Weyl chamber. The process stops when the particle hits one of the walls. One then computes the probability of going from an initial position $\mu_{i}$ to a final position $\lambda_{i}$ staying always within the chamber. This is given by [25]:

$$
p_{t, N}(\lambda, \mu)=\frac{1}{(2 \pi t)^{N / 2}} \mathrm{e}^{-\frac{|\lambda|^{2}+|\mu|^{2}}{2 t}} \operatorname{det}\left|\mathrm{e}^{\lambda_{i} \mu_{j} / t}\right|_{1 \leq i<j \leq N}
$$

The quickest way to make contact with Chern-Simons theory is to evaluate this amplitude in a very specific case: we take the same initial and final boundary conditions, i.e. $\mu=\lambda$, and an equal spacing condition, that is, $\lambda_{0 j}-\lambda_{0, j+1}=a$, where $a$ is the initial and final spacing between two neighboring movers. We compute the probability of a reunion after time $t$. Notice that, since the $\lambda$ 's also label highest weights of irreducible representations of $U(N)$, this boundary condition is labeled by the Weyl vector for a suitable choice of the overall scale. Now a straightforward computation yields:

$$
p_{t, N}\left(\lambda_{0}, \lambda_{0}\right)=\frac{1}{(2 \pi t)^{N / 2}} \prod_{k=1}^{N}\left(1-\mathrm{e}^{-k a^{2} / t}\right)^{N-k} .
$$

If one chooses units where $a^{2}=1$ and identifies $-\frac{1}{t}=g_{s}=\frac{2 \pi i}{k+N}$, then this is the Chern-Simons $S^{3} U(N)$ partition function. What about the explicit relationship between (3.1) and (1.3) ? In [24], we showed that the matrix model expression for the partition function of Chern-Simons on $S^{3}$ (1.3) corresponds to the extensivity property of probabilities:

$$
p_{t+t^{\prime}, r}(\rho, \rho)=\int[\mathrm{d} \lambda] p_{t, r}(\rho, \lambda) p_{t^{\prime}, r}(\lambda, \rho),
$$

where the range of integration is the same as in the matrix model. Now, the elementary but important step is to realize that (3.1) is also the density matrix description of a 
system of free fermions in one dimension at finite temperature $T$ and with harmonic confinement

$$
\begin{aligned}
\rho(y, x) & =C_{N} \operatorname{det}_{i j}\left\langle y_{i}\left|\exp \left(-\beta\left(\frac{\widehat{p}^{2}}{2 m}+\frac{1}{2} m \omega^{2} \widehat{x}^{2}\right)\right)\right| x_{j}\right\rangle \\
& =C_{N} \operatorname{det}_{i j}\left(\exp \left(-\frac{m \omega}{2 \hbar} \operatorname{coth}(\beta \hbar \omega)\left(y_{i}^{2}+x_{j}^{2}\right)+\frac{m \omega}{\hbar} \frac{y_{i} x_{j}}{\sinh (\beta \hbar \omega)}\right)\right)
\end{aligned}
$$

Indeed, this quantity is well-known from the early works that studied $c=1$ theory at finite temperature [26, 27, 28] (that is, with target space a circle instead of the line). Recall that string theory on a circle can be studied by a chain of matrices model that, in turn, was solved using a transfer matrix approach [26, 27] and the transfer matrix operator is precisely (3.4), which is also the propagator of the upside-down harmonic oscillator [26, 28]. In this correspondence we have that the string coupling constant is directly proportional to the temperature $g_{s} \sim T \sim 1 / t$. Note that this relationship is opposite to the one that appears in the crystal melting picture [29].

Thus, the underlying system is in principle the same: $N$ one-dimensional free fermions harmonically confined at finite temperature. What is the difference between the ChernSimons theory and string theory on a circle ? The Chern-Simons partition function is given by the overlap between identical initial and final states after temporal evolution in Euclidean time 3

$$
Z_{\mathrm{CS}}\left(S^{3}\right)=p_{t, N}\left(\lambda_{0}, \lambda_{0}\right)=\rho\left(x_{0}, x_{0}\right)
$$

where $x_{0}$ denotes a very particular position configuration with the fermions equispaced with unit distance. On the other hand, the partition function of string theory on a circle

$$
Z=\operatorname{Tre}^{-\beta R H}=\sum_{a} \int\left\langle\phi_{a}|\exp (-\beta H)| \phi_{a}\right\rangle=\sum_{a} \rho\left(x_{a}, x_{a}\right),
$$

implies the sum over all states.

In what follows, we shall discuss the free fermion at finite $\mathrm{T}$ behavior directly from the one matrix model formulation (1.3), (1.4) and from the point of view of exactly solvable models. Before proceeding, let us point out that the probability distribution (3.1) has also been considered in a very different setting in random matrix theory. More precisely, in the study of critical statistics [30]. The term critical statistics denotes a statistics of eigenvalues that interpolates between the Wigner-Dyson statistics [9] (typical of Gaussian matrix models and the one that is relevant in the metallic phase of a

\footnotetext{
${ }^{3}$ Euclidean time $t$ corresponds to the inverse of the string coupling constant $g_{s}$ and to the radius of the cylinder in the Brownian motion description (see the Conclusions).
} 
disordered system) and uncorrelated Poissonian statistics (insulating regime). Several matrix models in condensed matter physics have been introduced that incorporate this critical statistic, in an attempt to describe the metal to insulator transition in disordered systems. One of these models is the Moshe-Neuberger-Shapiro model, defined by 31

$$
\mathcal{P}_{U}(H) \mathrm{d}^{N^{2}} H=C_{N} \exp \left(-\operatorname{Tr} H^{2}-b \operatorname{Tr}\left([U, H][U, H]^{\dagger}\right)\right) \mathrm{d}^{N^{2}} H,
$$

where $U$ is an unitary matrix and $U=V^{\dagger} D V$ with $D_{i j}=\delta_{i j} \mathrm{e}^{i \theta_{i}}$. The idea of [31] is that $U$ defines a preferred basis with the $b$-dependent term, trying to align the Hermitian matrix $H$ with $U$ and hence leading to a preference for the basis $V$. Even after averaging over $U(N)$ with the invariant $U(N)$ Haar measure (which restores unitary invariance of the model) one obtains different statistics from the traditional Wigner-Dyson statistics. Note that the $b$-dependent term is of the same type as the one that naturally appears in matrix quantum mechanics [27]

$$
\operatorname{Tr}(\dot{\Phi})^{2}=\operatorname{Tr}\left[\dot{\Lambda}^{2}+[\Lambda, A][\Lambda, A]\right]
$$

with the matrix $\Phi(t)=\Omega(t) \Lambda(t) \Omega(t)$ with $\Omega$ unitary and $A(t)=\dot{\Omega} \Omega^{\dagger}$ a pure gauge field. This average over $U(N)$, as with the $c=1$ matrix model [27], is done using the Harish-Chandra-Izykson-Zuber integral, obtaining [31]

$$
P\left(x_{1}, \ldots, x_{N}\right)=\operatorname{det}_{i j}\left(\exp \left(-\left(b+\frac{1}{2}\right)\left(x_{i}^{2}+x_{j}^{2}\right)+2 b x_{i} x_{j}\right)\right)
$$

which is again (3.1) or, equivalently, (3.4) only that already in the diagonal representation. So, it is a particular case of (3.1) or (3.4), but the one relevant in Chern-Simons theory. The connection of this last expression with free fermions at finite temperature is also made in [31]. It is also worth to mention that one recurrent topic in many of the works that discuss the different random matrix models of critical statistics is to try to explain the fact that seemingly different matrix models, in particular (3.7) and (1.4) lead to the same statistics (their two-point correlation kernel, that we shall discuss below, coincide). The explanation, as we have seen above, is essentially contained in [24], as the matrix model representation comes from expressing the density probability as the composition of two processes, using an intermediate step to finally arrive at the departure point (3.3) .

Now, as stated above, the connection between the Chern-Simons matrix models and free fermions at finite temperature can also be seen from the properties of the 
corresponding one-matrix model (1.3), (1.4). The property is actually valid for $q$ deformed matrix model in general and for the Chern-Simons matrix model in particular. Recall that free fermions in one dimension are characterized by a first-order order coherence function:

$$
g(\vec{r})=\left\langle\widehat{\psi}^{\top}(r) \widehat{\psi}(0)\right\rangle=\frac{\sin \left(k_{0} r\right)}{\pi r},
$$

which is the two-point correlation of an Hermitian matrix model:

$$
K(x, y)=\frac{\sin (\pi(x-y))}{(x-y)}
$$

the famous sine kernel, describing the bulk correlations of a Hermitian Gaussian matrix model [9]. In the finite temperature case, it was found in [32] (see [33] for a modern and direct treatment) that $(3.10)$ is replaced by:

$$
g(\vec{r})=\frac{\delta k_{0} \sin k_{0} r}{\sinh \left(\pi r \delta k_{0}\right)}
$$

where $\delta k_{0}$ is the momentum width where the Fermi surface is no longer infinitely sharp, but has a smooth variation with an energy width of $k_{B} T$. More precisely, we have:

$$
\delta k_{0}=\frac{m k_{B} T}{\hbar^{2} k_{0}}
$$

It turns out that the two-point kernel of Hermitian random matrix ensembles with a $q$-deformed weight (for $\mathrm{e}^{-\pi^{2} / a} \ll 1$ ) 30 ]

$$
K(x, y)=\frac{a \sin (\pi(x-y))}{\sinh (a \pi(x-y))}, \text { where } a:=\frac{1}{2} \log \frac{1}{q}=\frac{g_{s}}{2},
$$

which is (3.12) with the $a$-parameter denoting the momentum width $\delta k_{0}$ and hence $g_{s} \sim T$ as above. The zero temperature limit implies $g_{s} \rightarrow 0$ and consequently $q \rightarrow 1$. This is the limit where the model goes to the Gaussian (GUE) universality class, which has a free fermion at zero temperature description, so we have a consistent result. The opposite limit $q \rightarrow 0$ (temperature tending to infinite) is less studied and will be discussed elsewhere (see the Conclusions). Note that finite temperature leads to a decrease of the correlations as the two-point function now decreases exponentially. The model (1.3) is of course more fluctuating than an ordinary Wigner-Dyson ensemble (a Gaussian model for example), with a higher level of repulsion between eigenvalues, and hence there is less correlation between particles, as they prefer to stay further away from each other. This can be made more precise at the level of the Hamiltonian, by recalling the results of the first Section. That is to say, it can be explained through 
additional interactions responsible for this temperature effect, that shows up as an strengthened repulsion at the level of the eigenvalue distribution. Let us show and briefly discuss here the explicit form of this additional interaction, that lead to the decrease of correlations typical of finite temperature effects on a system of fermions. In the Chern-Simons case we have seen that

$H=-\sum_{i=i}^{N} \frac{\partial^{2}}{\partial x_{i}^{2}}+\frac{1}{g_{s}^{2}} \sum_{i=1}^{N} x_{i}^{2}+\frac{m}{g_{s}} \sum_{i<j}\left(x_{i}-x_{j}\right) \operatorname{coth}\left(\frac{x_{i}-x_{j}}{2}\right)+\frac{m(m-1)}{2} \sum_{i<j} \frac{1}{\sinh ^{2}\left(\frac{x_{i}-x_{j}}{2}\right)}$,

is the Hamiltonian whose ground-state is the Chern-Simons eigenvalue distribution (1.3)

$$
H \Psi_{0}=\mathrm{E}_{0} \Psi_{0}, \quad \Psi_{0}\left(x_{1}, \ldots, x_{N}\right)=\prod_{i=1}^{N} \mathrm{e}^{-x_{i}^{2} / 2 g_{s}} \prod_{i<j}\left(2 \sinh \frac{x_{i}-x_{j}}{2}\right)^{m} .
$$

Consider the case of a Hermitian matrix model $m=1$ (the probability distribution of the matrix model is interpreted as the square of the wavefunction), that leads to the free fermion case in the Gaussian/Calogero model case. We see that even in this case a two-body correlation term survives

$$
V\left(x_{i}-x_{j}\right)=\frac{1}{g_{s}} \sum_{i<j}\left(x_{i}-x_{j}\right) \operatorname{coth}\left(\frac{x_{i}-x_{j}}{2}\right) .
$$

This is the term responsible for the departure with the usual free fermion at zero temperature behavior. Note how close is this interaction to be a Coulomb potential, as for a large separation between particles we have $V(x-x)=\left|x_{i}-x_{j}\right|$. Thus, it is the Coulomb potential in one-dimension, but modified at small separations with essentially a constant term (instead of going to 0 , as the Coulomb potential does). This result makes precise the observation in [34], where it is argued that the temperature effect can be described by additional interaction terms. Note also that this last result leads to an equivalent interpretation in terms of $\mathrm{N}$ fermions in one dimension with harmonic confinement, and two-body interactions described by (3.17) .

\section{Conclusions and Outlook}

We have seen that Chern-Simons partition functions can be obtained in two different ways: 
(i) As the norm of $\left|\Psi_{0}\right|^{2}$ as is typical of Chern-Simons theory $Z_{\mathrm{CS}}\left(S^{3}\right)=\left\langle\Psi_{0} \mid \Psi_{0}\right\rangle$, but with the $N$-body wavefunctions instead of wavefunctionals.

(ii) Specifying a very particular configuration where the fermions are equispaced as described in Section 3.

In the first Section, we explained that the model (2.1) describes a quasi-one dimensional Coulomb plasma, since it lives in $1 D$ but the repulsion between eigenvalues term $\left(V\left(x_{i}-x_{j}\right)=\ln \sinh \left(x_{i}-x_{j}\right)\right.$ in the Coulomb gas picture) is the Coulomb potential between two charges on the surface of a cylinder. This coincides with the non-intersecting Brownian motion description, as the returning condition for the $N$ walkers on the line implies that, in Euclidean time (as is the case of the matrix model description $-\frac{1}{t}=g_{s}$ ), we have $N$ non-intersecting Brownian motions on a cylinder, with the radial direction of the cylinder being the Euclidean time. Thus, the returning condition, essential to obtain the Chern-Simons partition function as we have seen, leads to the finite temperature result because it makes the Euclidean time to be compactified on a circle. This cylindrical geometry also emerges in the $D$-brane derivation of the matrix model [18].

Regarding the exactly solvable model, not only the connection with Chern-Simons theory is new but also the model has been poorly studied in itself. Hence, there are several open problems. For example, integrability of the model is an open problem and to find a Lax pair, provided the model is integrable, is a possible concrete task. It may also be of interest to study excited states of the model (something well-known for the Calogero model, and that involves the appearance of Jack polynomials, that comprise Schur functions), and see if they are related with states of the type (2.15) with $P=Q=$ 1 or, equivalently, with (2.19). In general, we believe that the appearance in ChernSimons theory of exactly solvable models that are so related to the celebrated Calogero and Sutherland models (see the introduction and for details on their relationship) is an interesting result that deserves further attention.

Besides topological strings, where Chern-Simons matrix models have already proved to be of interest, the models involved may play a direct role in condensed-matter physics as well. For example, it is immediate to identify (2.1) as the model that appears in a one-dimensional representation of a Laughlin state on a cylinder. An even more direct application has to do with the fact that other exactly solvable models with $F(x-y) \neq 0$ may be also of relevance in the study of Bose gases, as a generalization of the celebrated Lieb-Liniger model [35] and that these models are directly related to the Chern-Simons matrix model discussed here, in the limit where the cylindrical 
geometry above discussed is very thin.

Other physical applications besides topological strings, quantum Hall effect and Bose gas models, may have to do with the fact that quantum topological invariants play a role in the characterization of topological order. In the recent works [36], we find a connection between topological order and quantum dimensions. In general, a rich interplay between quantum computation, Chern-Simons theory and condensed matter physics has recently emerged. It would be certainly interesting if the many-body description of Chern-Simons theory and its topological invariants presented here could be employed in the context of topological quantum computation [37].

Acknowledgements. The author is grateful to Eduardo Fradkin, Jaume Gomis, Sebastian de Haro and Marcos Mariño for comments and correspondence.

\section{References}

[1] E. Witten, "Quantum field theory and the Jones polynomial," Commun. Math. Phys. 121, 351 (1989). 1, 2, 6

[2] E. Fradkin, Field theories of condensed matter systems, Addison-Wesley (1998). 1

[3] A. Neitzke and C. Vafa, "Topological strings and their physical applications," arXiv:hep-th/0410178; M. Mariño, "Chern-Simons theory and topological strings," Rev. Mod. Phys. 77 (2005) 675 arXiv:hep-th/0406005. 1, 7

[4] M. Mariño, "Chern-Simons theory, matrix integrals, and perturbative three-manifold invariants", Commun. Math. Phys. 253, 25 (2004), arXiv:hep-th/0207096. 1, 2, 5, 6, 8

[5] M. Tierz, "Soft matrix models and Chern-Simons partition functions," Mod. Phys. Lett. A 19, 1365 (2004) arXiv:hep-th/0212128, 1, 2, 5, , 8

[6] L. Rozansky, "A large $k$ asymptotics of Witten's invariant of Seifert manifolds," arXiv:hep-th/9303099, Commun. Math. Phys. 171 (1995) 279. "A contribution of the trivial connection to Jones polynomial and Witten's invariant of 3-D manifolds. 1" arXiv:hep-th/9401061, Commun. Math. Phys. 175 (1996) 275. "Residue formulas for the large $k$ asymptotics of Witten's invariants of Seifert manifolds: The case of $S U(2)$," arXiv:hep-th/9412075, Commun. Math. Phys. 178 (1996) 27. 1 
[7] R. J. Lawrence, "Asymptotic expansions of Witten-Reshetikhin-Turaev invariants for some simple 3-manifolds," J. Math. Phys. 36 (1995) 6106. R. Lawrence and L. Rozansky, "Witten-Reshetikhin-Turaev invariants of Seifert manifolds," Comm. Math. Phys. 205 (1999) 287. 1

[8] C. Beasley and E. Witten, "Non-abelian localization for Chern-Simons theory," J. Diff. Geom. 70, 183 (2005) arXiv:hep-th/0503126. 2

[9] M.L. Mehta, Random Matrices. Second edition. Academic Press 1991. 2, 10, 12

[10] S. de Haro and M. Tierz, "Discrete and oscillatory matrix models in Chern-Simons theory," Nucl. Phys. B 731 (2005) 225 arXiv:hep-th/0501123. 2, 5

[11] P.J. Forrester, "Vicious random walkers in the limit of a large number of walkers", J. Stat. Phys. 56, 767 (1989). 2

[12] V.I. Inozemtsev and D.V. Mescheryakov, "On ground-state wavefunctions for Sutherland-Calogero systems in an external field", Phys. Lett. A 106,101 (1984). 3

[13] T. Koprucki and H.J. Wagner, "New exact ground states for one-dimensional quantum many-body systems," J. Stat. Phys. 100, 779 (2000). 3, , 4

[14] P. J. Forrester, "Properties of an exact crystalline many-body ground state", J. Stat. Phys. 76, 331 (1994). 3, 5

[15] F.E. Harris and H.J. Monkhorst, "Electronic-Structure Studies of Solids. I. Fourier Representation Method for Madelung Sums ", Phys. Rev. B 2, 4400 (1970). 5

[16] Y. Dolivet and M. Tierz, "Chern-Simons matrix models and Stieltjes-Wigert polynomials," J. Math. Phys. 48, 023507 (2007) arXiv:hep-th/0609167, 6, 7

[17] M. Mariño, Personal communication 6

[18] M. Aganagic, A. Klemm, M. Mariño and C. Vafa, "Matrix model as a mirror of Chern-Simons theory," JHEP 0402, 010 (2004), arXiv:hep-th/0211098, 7, 14

[19] R. Dijkgraaf and C. Vafa, " $\mathrm{N}=1$ supersymmetry, deconstruction, and bosonic gauge theories," arXiv:hep-th/0302011. 8 
[20] M. R. Douglas, "Chern-Simons-Witten theory as a topological Fermi liquid," arXiv:hep-th/9403119. 8

[21] D. Ghoshal and C. Vafa, "C $=1$ String As The Topological Theory Of The Conifold," Nucl. Phys. B 453, 121 (1995) arXiv:hep-th/9506122, 8

[22] S. Mukhi, "Topological matrix models, Liouville matrix model and $\mathrm{c}=1$ string theory," arXiv:hep-th/0310287. 8

[23] Y. Nakayama, "Liouville field theory: A decade after the revolution," Int. J. Mod. Phys. A 19, 2771 (2004) arXiv:hep-th/0402009, 9

[24] S. de Haro and M. Tierz, "Brownian motion, Chern-Simons theory, and 2d YangMills," Phys.Lett. B 601, 201 (2004) arXiv:hep-th/0406093 S. de Haro, "ChernSimons theory, 2d Yang-Mills, and Lie algebra wanderers," Nucl. Phys. B 730,312 (2005) arXiv:hep-th/0412110. 9, 11

[25] M. E. Fisher, "Walks, Walls, Wetting, and Melting", J. Stat. Phys. 34, 667 (1984); D. A. Huse and M. E. Fisher, "Commesurate Melting, Domain Walls, and Dislocation”, Phys. Rev. B29, 239 (1984). 9

[26] D. J. Gross and I. R. Klebanov, "One-dimensional string theory on a circle," Nucl. Phys. B 344, 475 (1990). 10

[27] D. J. Gross and I. R. Klebanov, "Vortices and the nonsinglet sector of the $\mathrm{c}=1$ matrix model," Nucl. Phys. B 354, 459 (1991). 10, 11

[28] D. Boulatov and V. Kazakov, "One-Dimensional String Theory With Vortices As The Upside Down Matrix Oscillator," Int. J. Mod. Phys. A 8, 809 (1993) arXiv:hep-th/0012228. 10

[29] A. Okounkov, N. Reshetikhin and C. Vafa, "Quantum Calabi-Yau and classical crystals," arXiv:hep-th/0309208. 10

[30] K.A. Muttalib, Y. Chen, M.E.H. Ismail, and V.N. Nicopoulos, "New family of unitary random matrices," Phys. Rev. Lett. 71 (1993) 471; C. Canali and V. Kravtsov, "Normalization sum rule and spontaneous breaking of $\mathrm{U}(\mathrm{N})$ invariance in random matrix ensembles," Phys. Rev. E51 (1995) R5185; V.E. Kravtsov and K.A. Muttalib, "New Class of Random Matrix Ensembles with Multifractal Eigenvectors", Phys. Rev. Lett. 79 (1997) 1913. 10, 12 
[31] M. Moshe, H. Neuberger and B. Shapiro, "A Generalized ensemble of random matrices," Phys. Rev. Lett. 73, 1497 (1994). 11

[32] K.B. Efetov and A.I. Larkin, "Correlation functions in one-dimensional systems with a strong interaction", Sov. Phys. JETP 42, 390 (1976). 12

[33] Y. Castin, "Basic theory tools for degenerate Fermi gases", Proceedings of the Enrico Fermi Varenna School on Fermi gases (2006), arXiv:cond-mat/0612613. 12

[34] Y. Chen and K. A. Muttalib, "Crossover from Fermi Liquid to Non-Fermi Liquid Behavior in a Solvable One-Dimensional Model," J. Phys. Cond. Matt. 6, L293 (1994), arXiv:cond-mat/9311003 13

[35] M. Tierz, In preparation 14

[36] M. Levin and X.G. Wen, "Detecting order in a gound state wave function," Phys. Rev. Lett., 96, 110405 (2006) arXiv:cond-mat/0510613; A. Kitaev and J. Preskill, "Topological entanglement entropy," Phys. Rev. Lett. 96, 110404 (2006) arXiv:hep-th/0510092. 15.

[37] M. Freedman, M. Larsen, and Z. Wang, "A modular functor which is universal for quantum computation," Comm. Math. Phys., 227 605-622 (2002); M. Freedman, A. Kitaev, M. Larsen, and Z. Wang, "Topological quantum computation," Bull. Am. Math. Soc. 40, 31 (2003); A. Yu. Kitaev, "Fault-tolerant quantum computation by anyons." Ann. Phys., 303, 2-30 (2003). 